\title{
Callosal Contributions to Simultaneous Bimanual Finger Movements
}

\author{
Laura Bonzano, ${ }^{1,2}$ Andrea Tacchino, ${ }^{3}$ Luca Roccatagliata, ${ }^{1,2}$ Giovanni Abbruzzese, ${ }^{1}$ Giovanni Luigi Mancardi, ${ }^{1,2}$ and \\ Marco Bove ${ }^{3}$ \\ ${ }^{1}$ Department of Neurosciences, Ophthalmology, and Genetics, ${ }^{2}$ Magnetic Resonance Research Centre on Nervous System Diseases, and ${ }^{3}$ Department of \\ Experimental Medicine, Section of Human Physiology, University of Genoa, 16132 Genoa, Italy
}

Corpus callosum (CC) is involved in the performance of bimanual motor tasks. We asked whether its functional role could be investigated by combining a motor behavioral study on bimanual movements in multiple sclerosis (MS) patients with a quantitative magnetic resonance diffusion tensor imaging (DTI) analysis of CC, which is shown to be damaged in this disease. MS patients and normal subjects were asked to perform sequences of bimanual finger opposition movements at different metronome rates; then we explored the structural integrity of CC by means of DTI. Significant differences in motor performance, mainly referred to timing accuracy, were observed between MS patients and control subjects. Bimanual motor coordination was impaired in MS patients as shown by the larger values of the interhand interval observed at all the tested metronome rates with respect to controls. Furthermore, DTI revealed a significant reduction of fractional anisotropy (FA), indicative of microstructural tissue damage, in the CC of MS patients. By correlating the mean FA values with the different motor behavior parameters, we found that the degree of damage in the anterior callosal portions mainly influences the bimanual coordination and, in particular, the movement phase preceding the finger touch. Finally, the described approach, which correlates quantitative measures of tissue damage obtained by advanced magnetic resonance imaging tools with appropriate behavioral measurements, may help the exploration of different aspects of motor performance impairment attributable to the disease.

Key words: corpus callosum; finger opposition movement; bimanual coordination; MRI; diffusion tensor; multiple sclerosis

\section{Introduction}

Injury to corpus callosum (CC) impairs interhemispheric interactions that are important for bimanual motor performance as demonstrated by studies on patients who underwent partial or complete callosotomy (Geschwind and Kaplan, 1962; Preilowski, 1972; Jeeves et al., 1979; Eliassen et al., 2000; Serrien et al., 2001; Kennerley et al., 2002; Caille et al., 2005; Sternad et al., 2007). Additionally, CC pathology in patients affected by multiple sclerosis (MS) has been described (Evangelou et al., 2000) and shown to be associated with an alteration in the performance of tasks that require transfer of sensory information between cerebral hemispheres (Schnider et al., 1993), an impairment in finger localization and a decreased speed on an alternate finger-tapping task (Pelletier et al., 1992, 1993), a disruption of interhemispheric integration of motor information (Larson et al., 2002).

Although up to now surgical section of CC has created an exceptional condition to study the role of CC in bimanual coordination, here we propose an approach based on the correlation of data emerging from a motor behavioral study on bimanual

\footnotetext{
Received Sept. 6, 2007; revised Feb. 6, 2008; accepted Feb. 7, 2008.

This work was partly supported by Italian Multiple Sclerosis Foundation Projects 2004/R/3 and 2007/R/16. Data were collected and processed with Glove Analyzer System (GAS) provided by eTT (Electronic Technology Team, Genoa, Italy).

Correspondence should be addressed to Dr. Marco Bove, Department of Experimental Medicine, Section of Human Physiology, Viale Benedetto XV 3, 16132 Genoa, Italy. E-mail: marco.bove@unige.it.

D01:10.1523/JNEUROSCI.4076-07.2008

Copyright $\odot 2008$ Society for Neuroscience $\quad$ 0270-6474/08/283227-07\$15.00/0
}

movements and from a quantitative analysis of magnetic resonance diffusion tensor imaging (DTI) in MS patients. DTI may constitute a promising tool to study the CC function (JohansenBerg et al., 2007), and it can be indicative of a gradation of damage in specific portions of the CC in these patients. In fact, it has been shown that DTI can explore different aspects related to MS pathology in the CC (Ge et al., 2004; Oh et al., 2004; Hasan et al., 2005), allowing the study of the magnitude and directionality of water diffusion along white matter tracts, the diffusion tensor reconstruction, and the definition of diffusion parameters such as fractional anisotropy (FA), mean diffusivity, and diffusion eigenvalues (Le Bihan et al., 2001; Ge et al., 2005).

The present study was designed to address a few intertwined questions. The first aim was to understand whether MS patients showed an impaired motor behavior with respect to normal subjects when performing repetitive bimanual finger opposition movements paced with a metronome at different rates. Particularly, we investigated bimanual finger movements because the coordination of the hands and fingers is likely to rely on communication through the $\mathrm{CC}$ to an even greater extent than proximal limb movements (Gazzaniga, 1966; Gazzaniga et al., 1967; Brinkman and Kuypers, 1972, 1973; Eliassen et al., 2000). Then, we explored with DTI the structural integrity of the whole CC and of five single regions of interest in which we divided the CC for the DTI analysis. Our ultimate issue was to investigate the neural basis of motor deficits influencing bimanual movements; to this aim, we investigated whether impairments in bimanual motor 
Table 1. Demographic and clinical features of MS patients

\begin{tabular}{lllllc}
\hline $\begin{array}{l}\text { Patient } \\
\text { number }\end{array}$ & Sex & $\begin{array}{l}\text { Age } \\
\text { (years) }\end{array}$ & $\begin{array}{l}\text { MSpheno- } \\
\text { type }\end{array}$ & $\begin{array}{l}\text { EDSS } \\
\text { (months) }\end{array}$ \\
\hline 1 & F & 32 & RR & 2.5 & 48 \\
2 & F & 37 & RR & 3.5 & 84 \\
3 & F & 37 & RR & 1.0 & 12 \\
4 & F & 40 & RR & 5.0 & 48 \\
5 & F & 51 & SP & 5.5 & 144 \\
6 & F & 39 & RR & 3.0 & 120 \\
7 & F & 40 & SP & 4.5 & 72 \\
8 & F & 41 & SP & 6.0 & 204 \\
9 & F & 42 & RR & 3.0 & 108 \\
10 & M & 35 & RR & 1.0 & 72 \\
11 & M & 33 & RR & 1.0 & 192 \\
12 & M & 28 & RR & 1.5 & 60 \\
13 & M & 38 & RR & 1.0 & 144 \\
14 & M & 30 & RR & 3.0 & 84 \\
\hline
\end{tabular}

EDSS, Expanded disability status scale; $R R$, relapsing remitting; $S P$, secondary progressive; $F$, female; $M$, male.

behavior could be related to damages in the different identified regions of the CC in MS patients. Data from motor behavior analysis were thus combined with quantitative information obtained through DTI, correlating the degree of microstructural damage of segments of the CC with the different motor behavior parameters.

\section{Materials and Methods}

Subjects. Fourteen patients (nine females and five males; age range, 28-51 years; mean $\pm \mathrm{SD}, 37.4 \pm 5.8$ years; mean \pm SD disease duration, $99.4 \pm$ 55.8 months) affected by MS and 10 healthy volunteers (eight females and two males; age range, $26-47$ years; mean \pm SD, $33.4 \pm 6.0$ years) were included in this study. All subjects were naive to the specific purpose of this study. Informed consent was obtained according to our institution policy and to the Declaration of Helsinki. Inclusion criteria were the following: both sexes, age older than 18 years, and right-handed according to the Edinburgh Handness Inventory (Oldfield, 1971), and patients had definite MS as determined by Poser's criteria (Poser et al., 1983), with stable phase of the disease without relapses or modifications in neurological examination in the last 3 months. Exclusion criteria comprised the following: magnetic resonance imaging (MRI) contraindications (claustrophobia, pregnancy, and presence of a pacemaker and other metallic parts not magnetic resonance compatible), inability to comfortably perform simple finger-tapping movements at a frequency of $\sim 1 \mathrm{~Hz}$, and use of medications known to influence cerebellar function and/or muscle tone, such as antiepileptic drugs, benzodiazepine, antidepressants, Beta blockers, drugs for spasticity. All patients were clinically evaluated at the time of enrolment with the Kurtzke expanded disability status scale (Kurtzke, 1983). Clinical disability of MS patients ranged from mild to moderate as reported in Table 1.

Apparatus. Subjects wore a sensor-engineered glove (patent pending, number TO2005A00368, 31/05/2005) on both hands. Data were acquired at $1 \mathrm{kHz}$ (board 800008B-01; National Instruments, Austin, TX); custom-made software generated pacing signals and recorded the time of each tone and the time of each finger touch (Bove et al., 2007).

Behavioral paradigm. Subjects were asked to perform repetitive finger opposition movements of thumb to index, medium, ring, and little fingers with the two hands simultaneously and to pace the movements with a metronome tone delivered through isolation headphones. Three different rates were randomly presented $(1,1.5$, and $2 \mathrm{~Hz})$; the choice of this range of rates was based on the experimental evidence that MS patients were not fully compliant with movements faster than $2 \mathrm{~Hz}$. In fact, we were mainly interested in studying the motor behavior during the performance of correct sequences in which the percentage of number of errors was limited but meaningful as comparison parameter. Although these rates do not differ much from one another, it was shown that, from 1 to $2 \mathrm{~Hz}$, normal subjects began to significantly change their motor behavior during the execution of unimanual repetitive finger movements (Bove et al., 2007).

Subjects were instructed through a video that showed a hand executing the task three times, and then they practiced the task at their own pace. Training ended generally within $2 \mathrm{~min}$, when they were able to execute the task without errors. Then, they were seated in a comfortable chair in a quiet and darkened room. An eyes-closed paradigm was chosen to exclude possible confounding effects attributable to the integration of acoustic and visual information and to prevent MS patients from compensating for possible motor impairments by visual inspection. Testing session included three $45 \mathrm{~s}$ trials (one per rate). At the beginning of each block, three tones were given to signal the task pacing; subjects were instructed to listen to and to wait for these three tones and to start pacing their first movement with the fourth tone. A signal indicated the end of each block; blocks were separated by 2 min rest. Subjects were also instructed to stop a sequence and to quickly restart from the first finger when they made errors such as skip of a finger, a double touch to the same finger, and a touch of the thumb to two or more fingers at once.

Motor behavioral data analysis. To describe the different aspects of the studied motor task, data were processed with customized software, and a number of parameters were defined for the specific analysis. Bimanual coordination was studied by means of the interhand interval (IHI) to assess possible impairments already observed in patients with damages at the level of corpus callosum (Eliassen et al., 2000). For bimanual coordination, we meant when two hands are able to perform a bimanual motor task with a high level of synchronization; specifically in our case, it means synchronized movements of equivalent fingers in the two hands. Thus, the absolute value of the timing difference between these movements was chosen to demonstrate that the two hands do not make the same movement exactly in a simultaneous way. According to this definition, the larger is the IHI absolute value, the more severe is the impairment in bimanual coordination. Particularly, we identified two measures of IHI: $\mathrm{IHI}_{\text {onset }}$, defined as the absolute time difference between the touch onset occurring in the left hand and the corresponding touch in the right hand, and $\mathrm{IHI}_{\text {offset }}$, defined as the absolute time difference between the finger touch offset occurring in the two hands.

Finally, to investigate the presence of possible motor impairments of MS patients in the performance of finger opposition movement sequences, we studied both the finger-touching phase and the transition phase from a finger to the successive one in the sequence. These two phases correspond, respectively, to the touch duration (TD), computed as the contact time between thumb and another finger, and the intertapping interval (ITI), the time between the end of the contact of thumb and another finger and the beginning of the successive contact. Furthermore, temporal and spatial accuracy was investigated by evaluating the relative timing and the errors made during the motor sequence performance. Relative timing was defined as the time between the touch onset and the corresponding acoustic cue: it was negative when the touch preceded the metronome tone and positive when the touch followed the metronome tone. For each trial, we computed the percentage of errors over the total number of touches made during the motor task, and the incorrect sequences were discarded from additional analysis.

Magnetic resonance imaging protocol. MRI was performed on a 1.5 tesla MR system (Signa Excite; GE Medical Systems, Milwaukee, WI). Each subject underwent brain MRI examination including coronal $\mathrm{T}_{1}$ weighted three-dimensional spoiled gradient recalled echo imaging [slice thickness, $1.5 \mathrm{~mm}$; repetition time (TR), $28 \mathrm{~ms}$; echo time (TE), $6 \mathrm{~ms}$; flip angle, $20^{\circ}$; field of view $(\mathrm{FOV}), 260 \mathrm{~mm}$; matrix, $256 \times 256$ ], axial proton density $/ \mathrm{T}_{2}$-weighted imaging (slice thickness, $3 \mathrm{~mm}$; TR, $3000 \mathrm{~ms}$; TE, 16.1-96.8 ms; flip angle, $90^{\circ}$; FOV, $250 \mathrm{~mm}$; matrix, $256 \times 256$ ), and, in patients only, $\mathrm{T}_{1}$-weighted images after gadolinium chelate administration at the dosage of $0.1 \mathrm{mmol} / \mathrm{kg}$. Diffusion tensor imaging was performed by using single-shot spin-echo echoplanar sequences, with diffusion gradients applied in 15 noncollinear directions $\left(b=1000 \mathrm{~s} / \mathrm{mm}^{2}\right)$ and two baseline acquisitions without diffusion gradients (slice thickness, $2 \mathrm{~mm}$; TR, 16,000 ms; TE, $105 \mathrm{~ms}$; flip angle, 90 FOV, $240 \mathrm{~mm}$; matrix, $256 \times 256$ ). All the series covered the whole brain and were acquired without gap between slices.

Magnetic resonance DTI analysis. Data obtained with diffusion tensor 


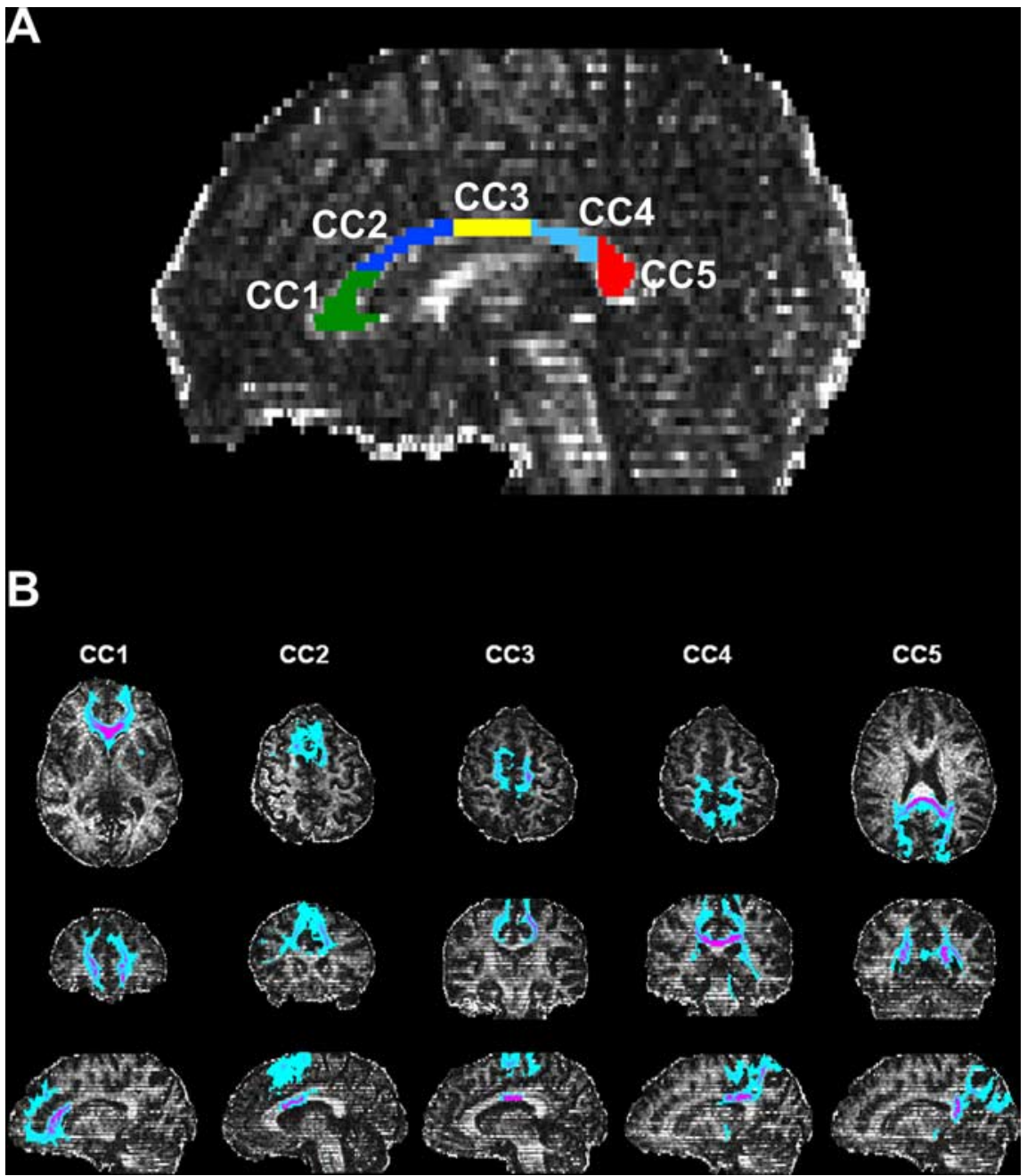

Figure 1. Representative fractional anisotropy map of a control subject with the subdivision of corpus callosum in five regions of interest $(\mathrm{CC} 1-\mathrm{C}(5)(\boldsymbol{A})$ and paths connecting callosal regions with cortical areas obtained by probabilistic tractography and displayed in axial, coronal, and sagittal plane $(\boldsymbol{B})$.

imaging were processed by using FDT, a software tool for analysis of diffusion-weighted images, part of FSL (Smith et al., 2004). Highdiffusion anisotropy is primarily expected in the CC (Chepuri et al., 2002), because most axonal fibers are directed horizontally. Furthermore, the degree of diffusion anisotropy increases with normal myelination processes and with organization of axons during development (Neil et al., 1998), and CC is a highly organized and myelinated structure (Aboitiz et al., 1992). For these reasons, FA is particularly indicated to study pathological processes involving the CC; a reduction of FA in the CC of MS patients has been shown previously (Ge et al., 2004; Oh et al., 2004; Hasan et al., 2005). Thus, we obtained FA parametric maps to quantify damage along the corpus callosum.

Subregions of the CC were selected similarly to a previously described method that used a geometrical scheme to divide the CC into five parts on the basis of an "anterior-to-posterior" distance, measured on a curve from the tip of the rostrum to the tip of the splenium, with the first subregion accommodating the rostrum and the entire genu (de Lacoste et al., 1985). Regions of interest (ROIs) were drawn in the CC of each subject on the midsagittal plane of FA maps (Fig. $1 A$ ), excluding voxels at the edge to minimize possible partial-volume artifacts; the anatomic correspondence of the ROIs was visually checked on the $\mathrm{T}_{2}$-weighted baseline echoplanar images. One ROI (CC1) included the genu and the rostrum, another ROI (CC5) covered the splenium, and three ROIs (CC2, $\mathrm{CC} 3$, and CC4) were identified in the remaining portion of the CC, mainly represented by the body, subdividing the anterior-to-posterior distance into three parts.
Moreover, for control subjects, we complemented the anatomic subdivision of the $\mathrm{CC}$ with information derived from tractography, to reveal paths connecting callosal regions with cortical areas (Fig. $1 B$ ). To achieve this, each single ROI was used as seed mask for probabilistic tractography (Behrens et al., 2003). Tractography identifies white matter bundles mostly in agreement with classical anatomical knowledge even when technical limitations might lead to falsepositive or negative results attributable to noise, voxel contamination effects, and coexistence within a voxel of multiple fibers with complex architecture. Mean FA values were then calculated in the whole CC and within each ROI for all the enrolled subjects.

Statistics. The changes in $\mathrm{IHI}_{\text {onset }}$, $\mathrm{IHI}_{\text {offset' }}$, TD, ITI, relative timing, and the percentage of errors made during the bimanual motor task were subjected, separately, to ANOVA. Precisely, bimanual coordination parameters were separately analyzed by means of a one-way ANOVA with the variable group (MS patients and control subjects) used as between-subject factor and metronome rate $(1,1.5$, and $2 \mathrm{~Hz})$ as within-subject factor. Furthermore, the $\mathrm{IHI}_{\text {onset }}$ and $\mathrm{IHI}_{\text {offset }}$ were compared between them by means of a two-way ANOVA with group (MS patients and control subjects) as betweensubject factor and metronome rate $(1,1.5$, and 2 $\mathrm{Hz})$ and $\mathrm{IHI}\left(\mathrm{IHI}_{\text {onset }}, \mathrm{IHI}_{\text {offset }}\right)$ as withinsubject factors. For single-hand kinematics parameters (TD, ITI, relative timing, and percentage of errors), a two-way ANOVA was performed, separately for each parameter, with group (MS patients and control subjects) as between-subject factor and metronome rate ( 1 , 1.5 , and $2 \mathrm{~Hz}$ ) and hand (dominant and nondominant hand) as within-subject factors. When ANOVA gave a significant result $(p<$ $0.05)$, post hoc Newman-Keuls test was used to assess significant differences.

After assessing that FA values did not show a normal distribution by using the Shapiro-Wilk normality test, the comparisons to find out differences in the mean FA values both in the whole CC and within the single CC ROIs between MS patients and control subjects were performed by means of the nonparametric Mann-Whitney $U$ test. Then, Pearson's correlation coefficients were calculated between the FA in each ROI of the CC and the movement parameters for MS patients. Correlations were performed for each metronome rate; in addition, when the statistical analysis indicated that a specific motor behavior parameter was not significantly influenced by rate, an average on the values obtained for the three metronome rates was performed and then correlated with FA values of the CC ROIs.

Numerical data are mean \pm SE unless otherwise stated.

\section{Results}

\section{Motor behavior}

The analysis of motor behavior parameters was performed to assess any significant difference in the motor performance of bimanual finger movements between MS patients and control subjects. Bimanual coordination was impaired in MS patients as shown by the significantly larger values of $\mathrm{IHI}_{\text {onset }}$ and $\mathrm{IHI}_{\text {offset }}$ with respect to control subjects (Fig. 2 ) ( $\mathrm{IHI}_{\text {onset }}, F_{(1,22)}=4.9, p=$ $\left.0.036 ; \mathrm{IHI}_{\text {offset }}, F_{(1,22)}=4.6, p=0.043\right)$. In both groups, $\mathrm{IHI}_{\text {onset }}$ and $\mathrm{IHI}_{\text {offset }}$ did not significantly change their values with metronome rate $\left(\mathrm{IHI}_{\text {onset }}, F_{(1,22)}=2.7, p=0.075 ; \mathrm{IHI}_{\text {offset }}, F_{(1,22)}=1.1\right.$, $p=0.33)\left(\mathrm{IHI}_{\text {onset }}: 1 \mathrm{~Hz}, 44.7 \pm 8.1\right.$ vs $24 \pm 4.5 \mathrm{~ms} ; 1.5 \mathrm{~Hz}, 39.1 \pm$ 

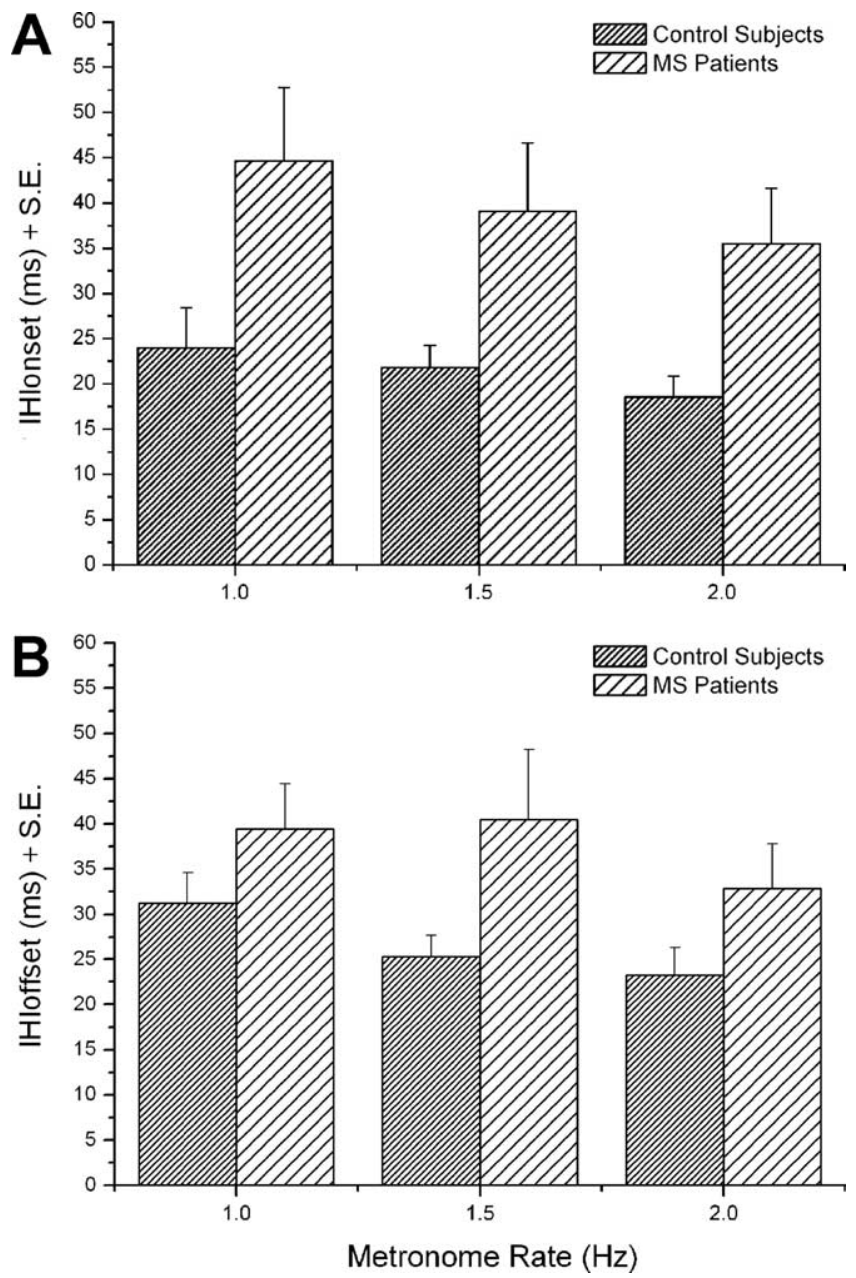

Figure 2. Control subjects and MS patients performing bimanual finger opposition movements at 1, 1.5, and $2 \mathrm{~Hz}$ : mean interhand interval onset $(\boldsymbol{A})$ and offset $(\boldsymbol{B})$ evaluated for the different metronome rates.

7.5 vs $21.8 \pm 2.5 \mathrm{~ms} ; 2 \mathrm{~Hz}, 35.5 \pm 6.1$ vs $18.5 \pm 2.3 \mathrm{~ms} ; \mathrm{IHI}_{\text {offset: }}$ : $1 \mathrm{~Hz}, 39.4 \pm 5$ vs $31.2 \pm 3.4 \mathrm{~ms} ; 1.5 \mathrm{~Hz}, 40.5 \pm 7.8$ vs $25.3 \pm 2.4$ ms; $2 \mathrm{~Hz}, 32.9 \pm 4.9$ vs $23.3 \pm 3.1 \mathrm{~ms})$. Furthermore, by comparing $\mathrm{IHI}_{\text {onset }}$ and $\mathrm{IHI}_{\text {offset }}$, we did not find any significant difference between them $\left(F_{(1,44)}=0.54, p=0.47\right)$. This finding suggests that MS patients had a similar impairment in coordinating the finger movements of the two hands in both the phase preceding the finger touch and the phase of shifting from a finger to the successive one of the sequence.

In addition, to describe the hand movement in its entirety, we evaluated the kinematic parameters that characterize the single hand movements during the bimanual finger motor task, such as touch duration, intertapping interval, relative timing, and percentage of errors made in the motor sequences. ANOVA indicated that, in both groups, there was no significant difference among all the motor behavior parameters between dominant and nondominant hand. Therefore, in the following, results dealing with touch duration, intertapping interval, relative timing, and percentage of errors refer only to the dominant hand.

Overall, TD was significantly influenced by metronome rate in both groups $\left(F_{(2,44)}=14,214, p=0.00002\right)$. However, MS patients showed significantly larger TD values with respect to control subjects $\left(F_{(1,22)}=4.8, p=0.04\right)$ for all the rates $(1 \mathrm{~Hz}$, $366.7 \pm 41.4$ vs $249.9 \pm 12.8 \mathrm{~ms} ; 1.5 \mathrm{~Hz}, 305.3 \pm 32.9$ vs $235.6 \pm$ $21.9 \mathrm{~ms} ; 2 \mathrm{~Hz}, 261.4 \pm 27.8$ vs $202.7 \pm 14.8 \mathrm{~ms}$ ).

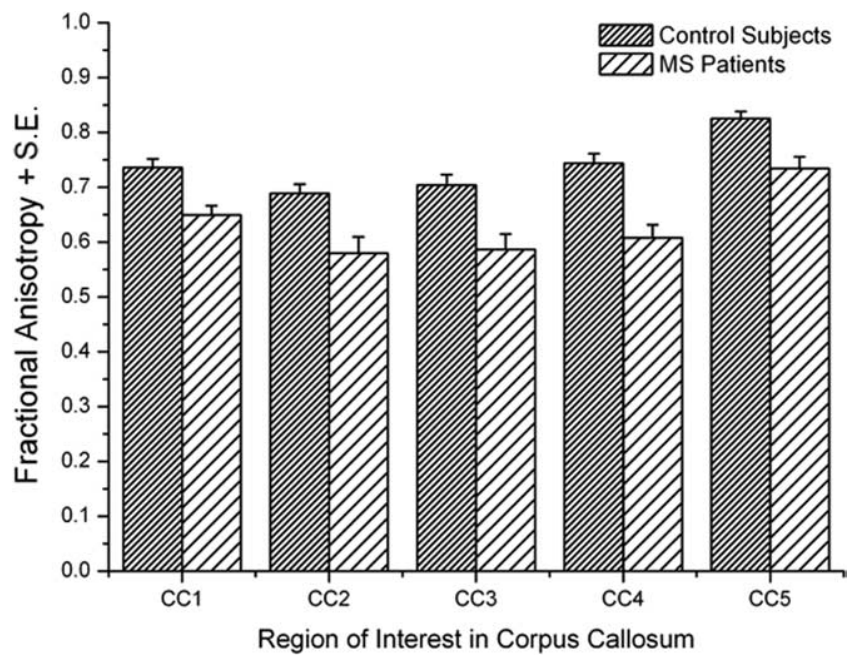

Figure 3. Mean fractional anisotropy in the single regions of interest in the corpus callosum for control subjects and MS patients.

Conversely, intertapping interval did not significantly differ between MS patients and control subjects $\left(F_{(1,22)}=0.02, p=\right.$ $0.89)$. We also found that, in control subjects, at the different rates, relative timing had a negative value of $\sim 80 \mathrm{~ms}$ with respect to the occurrence of the acoustic cue in agreement with other studies (Aschersleben, 2002; Bove et al., 2007). A $t$ test analysis on relative timing with respect to zero (set as the time when the metronome signal occurs) revealed that control subjects showed a significant negative asynchrony in finger movements with respect to the metronome signal ( $p$ always $<0.05$ at all the different rates). Notably, a significant reduction of this asynchrony was observed in MS patients with respect to controls $\left(F_{(1,22)}=4.49\right.$, $p=0.045)$, and it was significant for all the rates $(1 \mathrm{~Hz},-14 \pm$ 23.1 vs $-80 \pm 18.4 \mathrm{~ms}, p<0.05 ; 1.5 \mathrm{~Hz},-22.7 \pm 22.9$ vs $-75.8 \pm 24.6 \mathrm{~ms}, p<0.05 ; 2 \mathrm{~Hz},-6.6 \pm 9.8 \mathrm{vs}-74.7 \pm 19.7 \mathrm{~ms}$, $p<0.05)$. This reduction was so marked that, in these patients, because of the presence of several touches performed close to the metronome signal, we did not find any significant difference from zero that could allow us to identify negative or positive asynchronies from relative timing data (always $p>0.05$ ).

Spatial accuracy was evaluated by calculating the percentage of errors made by MS patients and control subjects at the different rates. As a result, a significant difference in the mean percentage of errors between the two groups was observed $\left(F_{(1,22)}=6.39\right.$, $p=0.02)$. However, post hoc analysis revealed that this difference was significant only at $2 \mathrm{~Hz}(18.4 \pm 2.6$ vs $6.4 \pm 2.3 \%, p<0.05)$.

\section{Diffusion tensor imaging}

The analysis of FA values obtained from diffusion tensor imaging was performed to assess any significant difference in the structural integrity of the corpus callosum between MS patients and control subjects. Particularly, we were interested in investigating the existence of tissue damage involving even single portions of the CC. Mean FA values were calculated for all the MS patients and control subjects in both the whole CC and each single identified ROI (Fig. 3).

An overall damage in the CC of MS patients was demonstrated by a statistically significant decrease in the mean FA value with respect to the control group $(0.63 \pm 0.02$ vs $0.74 \pm 0.01, U=$ $10.5, p=0.0005)$. Interestingly, Mann-Whitney $U$ test with Bonferroni's correction $(p<0.01)$ showed a significant reduction of FA in MS patients with respect to controls in each analyzed por- 
A

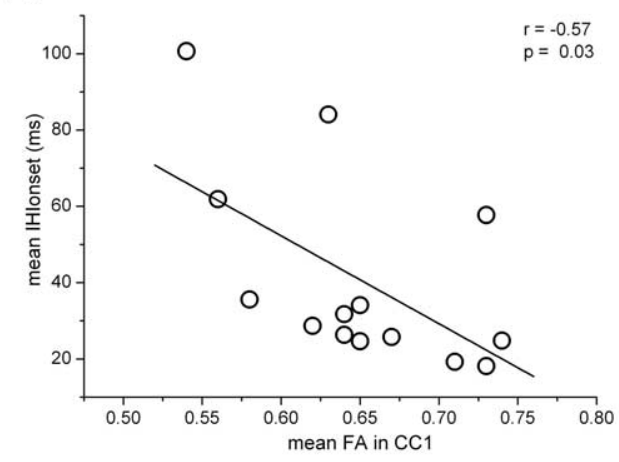

B

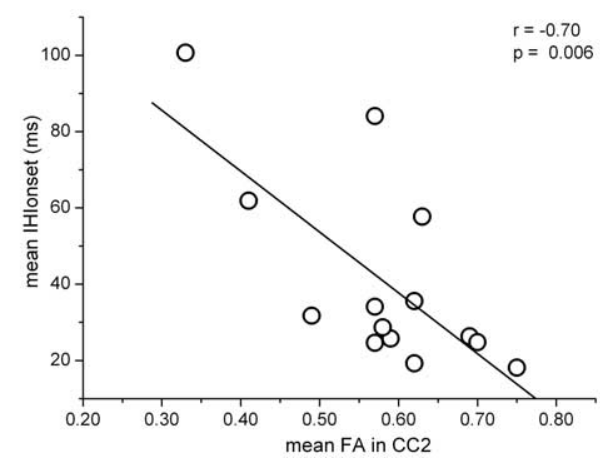

C

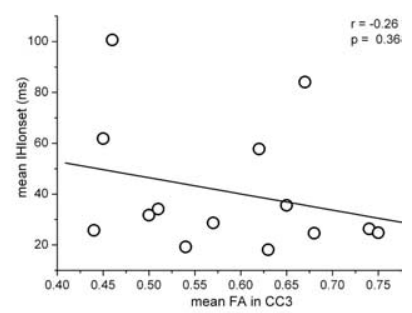

D

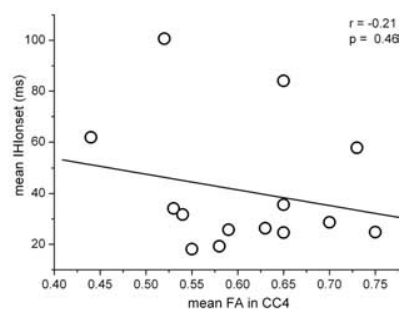

E

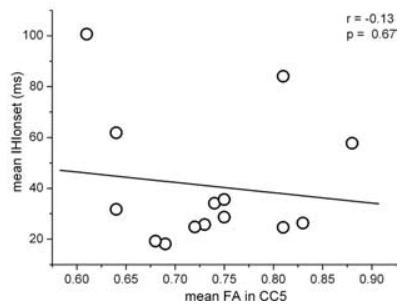

Figure 4. $\quad \mid \mathrm{HI}_{\text {onset }}$ averaged on the three metronome rates as function of mean FA in $\mathrm{CC} 1(\boldsymbol{A}), \mathrm{CC}(\boldsymbol{B}), \mathrm{CC} 3(\boldsymbol{C}), \mathrm{CC} 4(\boldsymbol{D})$, and CC5 $(\boldsymbol{E})$, with the corresponding linear fitting (solid line). Pearson's correlation coefficient $r$ and $p$ value are reported; as shown, $\mathrm{IHI}_{\text {onset }}$ correlated with FA only in CC1 and CC2.

tion of the CC $(\mathrm{CC} 1, U=18, p=0.002$; $\mathrm{CC} 2, U=23.5, p=$ 0.006; CC3, $U=25, p=0.008$; CC4, $U=13, p=0.0008$; CC5, $U=20.5, p=0.004)$.

\section{Motor behavior and DTI}

To study the role of corpus callosum in simultaneous bimanual finger movements, mean FA values within different ROIs along the CC of MS patients were correlated with their motor behavior parameters whose values were significantly different from those observed in control subjects. This analysis revealed a correlation of specific parameters with FA only in precise CC subregions. In particular, $\mathrm{IHI}_{\text {onset }}$ significantly increased in correspondence of an FA decrease for all the rates in both CC1 and CC2 (1 Hz: CC1, $r=-0.53, p<0.05$; CC2, $r=-0.74, p<0.001 ; 1.5 \mathrm{~Hz}: \mathrm{CC} 1, r=$ $-0.57, p<0.05$; CC2, $r=-0.73, p<0.001$; $2 \mathrm{~Hz}$ : CC1, $r=$ $-0.68, p<0.05$; CC2, $r=-0.58, p<0.05)$, whereas IHI $_{\text {offset }}$ was inversely correlated with FA only in CC1 and CC2 at $2 \mathrm{~Hz}(2 \mathrm{~Hz}$ : CC1, $r=-0.54, p<0.05$; CC2, $r=-0.60, p<0.05)$. Then, an increase of TD correlated with a reduction of FA in CC2 only for 1 and $1.5 \mathrm{~Hz}$ but not for $2 \mathrm{~Hz}(1 \mathrm{~Hz}$ : CC2, $r=-0.53, p<0.05 ; 1.5$ Hz: CC2, $r=-0.66, p<0.001)$, whereas relative timing showed a negative correlation with FA only in CC3 when the movements were paced by a metronome rate of 1.5 and $2 \mathrm{~Hz}(1.5 \mathrm{~Hz}$ : CC3, $r=$ $-0.58, p<0.05 ; 2 \mathrm{~Hz}$ : CC3, $r=-0.54, p<0.05)$. Besides, the percentage of errors did not correlate with any CC ROI.

Furthermore, because both $\mathrm{IHI}_{\text {onset }}$ and $\mathrm{IHI}_{\text {offset }}$ did not significantly differ among the three metronome rates, we averaged the $\mathrm{IHI}_{\text {onset }}$ and $\mathrm{IHI}_{\text {offset }}$ values on these rates and correlated them with the FA values in the different ROIs. The results confirmed that mean $\mathrm{IHI}_{\text {onset }}$ was linearly correlated with $\mathrm{FA}$ values only in CC1 and CC2 (Fig. 4), whereas no significant correlation was found for mean $\mathrm{IHI}_{\text {offset }}$ in any ROI of CC.

Analogously, relative timing was not influenced by metronome rate. Thus, values on the three rates were averaged and evaluated in relation to CC damage. As a result, no statistical significance was found in any CC ROI.

\section{Discussion}

Our behavioral results show significant differences in the performance of repetitive bimanual finger opposition movements between MS patients and control subjects. When investigating bimanual coordination by studying the interhand interval, we found that MS patients had an impairment in the coordination of bimanual finger movements because they showed larger $\mathrm{IHI}_{\text {onset }}$ and $\mathrm{IHI}_{\text {offset }}$ values than control subjects at all the tested rates. Similarly, in literature, MS patients were found to be slower than normal subjects when they performed the bimanual coordination test. Precisely, a deficit in bimanual motor coordination was observed only in those patients with abnormal cross-callosal-evoked potentials, evidence of inefficient callosal transmission, supporting the conclusion that deficits in bimanual motor coordination occur in MS and are related to callosal dysfunction (Larson et al., 2002). Impairments were also observed in both temporal and spatial parameters describing the single hand movement during the bimanual motor task execution, with predominance in the temporal ones. In fact, in MS patients, significantly larger TD values were observed with respect to control subjects at all the tested metronome rates, suggesting that MS patients need more time to evaluate the finger contact and recognize the touched finger before moving forward in the performance of the sequence.

As shown by smaller relative timing values, MS patients did not show the negative asynchrony observed in control subjects at all the tested rates. This is in contrast with a study showing that, when no movement-related feedback was available, deafferented patients revealed a stable timing pattern with large negative asynchronies, reflecting anticipatory timing (Stenneken et al., 2006). On the contrary, our results are in agreement with those obtained by studying patients with cerebral lesions resulting in somatosensory loss (Najenson et al., 1989; Stenneken et al., 2003; Takahashi and Reinkensmeyer, 2003). These findings point to the importance of unimpaired central processing loops for anticipatory timing. Thus, problems in timing observed in MS patients could be related to a sensorimotor impairment at the central level rather than to a simple problem of sensory transmission from periphery (Aschersleben, 2002).

The ultimate issue of this work was whether modifications in bimanual finger motor performance could correlate with microstructural changes in different regions of the corpus callosum in MS patients. To answer this question, we first assessed the presence of significant differences in the structural integrity of the CC between MS patients and control subjects; this was done by means of DTI, extracting the values of FA within the five different ROIs delineated along the CC. We found that MS patients had 
lower FA values than controls in both the whole CC and each of its analyzed portions. A DTI investigation of CC subregion integrity in MS has been reported in previous work, in which not all the analyzed segments of the CC were found to be involved by significant pathological alterations (Hasan et al., 2005). These discrepancies might be related to the fact that we adopted a different partition of the $\mathrm{CC}$ and that they studied patients without lesions detectable by conventional MRI to gain insight on the possible pathogenesis of occult MS-related damage in the normal-appearing white matter and its extension to the CC itself. Conversely, our patients were not selected by the absence or presence of $\mathrm{T}_{2}$-weighted lesions in the $\mathrm{CC}$ because our intent was to quantify with FA the CC damage related to MS independently from its detectability on conventional images and to correlate it with motor behavior parameters. Furthermore, as reported in previous DTI studies (Chepuri et al., 2002), we appreciated that, also in healthy subjects, FA values within CC subregions vary; these differences reveal the effects of fiber density, orientation, thickness of individual axons, and degree of myelination on water diffusion anisotropy. This heterogeneity in CC structure is related to the connection with functionally different cortical areas. To partition the CC, to identify the borders of CC subregions there are not precise anatomic landmarks, different methods have been used, among which the one proposed by de Lacoste et al. (1985). We divided the CC of each subject on the basis of this model; moreover, we estimated the connections from our ROIs to cortical areas by means of probabilistic tractography (Behrens et al., 2003) to visualize cortical projections from each delineated ROI. According to this model, CC1 should contain mainly fibers connecting with prefrontal regions, $\mathrm{CC} 2$ is the subregion of the $\mathrm{CC}$ that contains fibers projecting to premotor and supplementary motor areas, CC3 mainly includes fibers related to primary motor and sensory areas, whereas CC4 and CC5 have their projections on posterior parietal, temporal, and occipital areas.

Then, we correlated the FA values within the CC ROIs with the motor behavior parameters calculated at the different rates and, when possible, with their values averaged on the three rates; we obtained different results for the five ROIs of the CC in relation to the evaluated parameter. Interestingly, FA values evaluated in MS patients in $\mathrm{CC} 1$ and $\mathrm{CC} 2$ regions well correlated with $\mathrm{IHI}_{\text {onset }}$ at any rate and with the averaged value. This finding could indicate that the impairment in bimanual coordination observed in these patients may be attributable to difficulties in the information exchange between the prefrontal and motor areas of the two hemispheres. Notably, among the different functional properties of the prefrontal cortex, there is the temporal organization and control of complex behavioral strategies (Miller, 2000; Koechlin and Jubault, 2006). Besides, this strong correlation with FA was not found for $\mathrm{IHI}_{\text {offset }}$, which inversely correlated with FA in CC1 and CC2 only at $2 \mathrm{~Hz}$ and not when the values at the different rates were averaged and combined with FA values. This finding could suggest that the information necessary to coordinate the bimanual finger opposition movements is exchanged between the two cortical hemispheres only during the approaching phase to finger touch and not during the phase of shifting from a finger to the successive one of the sequence. As for $\mathrm{IHI}_{\text {offset }}$, the correlation of TD and relative timing with FA was not systematic for all the metronome rates. Particularly, the larger values of TD observed in MS patients with respect to controls correlated with the lower values of FA observed in CC2, but only at 1 and $1.5 \mathrm{~Hz}$. The alterations in relative timing found in MS patients well correlated with a reduced integrity of corpus callosum fibers in region CC3, only at high metronome rates. Furthermore, whereas the tempo- ral parameters of the repetitive bimanual finger movements were significantly influenced by the interhemispheric transfer of information, the percentage of errors showed only a slight difference in MS patients with respect to controls and no correlation with any region of the $\mathrm{CC}$ was found. A possible explanation is that the errors made during the performance of a finger movement sequence are related to faults in the motor programming generated at the level of the two separate hemispheres and not to a deficit in sensorimotor transmission between them.

All these findings emphasize that the effect of FA in corpus callosum on movement control is unique to bimanual coordination, and it mainly influences the coordination phase of movement preceding the finger touch.

We can conclude that anterior and body callosal connections are essential to perform temporally interdependent bimanual finger movements (Preilowski, 1972; Eliassen et al., 2000), and, even if the superior temporal gyrus communicates through the posterior corpus callosum (CC4 and/or CC5), this portion of corpus callosum seems not to be crucial in the coordination of acoustically paced bimanual finger opposition movements. This is in disagreement with a previous work showing the importance of posterior corpus callosum in bimanual coordination (Eliassen et al., 2000); however, whereas visual information was used in this experimental paradigm, in our study, subjects performed acoustically paced motor tasks without any visual facilitation. Furthermore, it was found that visual and visuospatial integration is disrupted by posterior callosotomy (Gazzaniga and Freedman, 1973; Sidtis et al., 1981; Volpe et al., 1982; Risse et al., 1989; Eliassen et al., 1999; Marzi et al., 1999), whereas timing coordination is altered by anterior callosotomy (Preilowski, 1972, 1975; Eliassen et al., 1999). Also, the evaluation of the crossed-uncrossed difference in simple reaction times with visual stimuli, to measure the interhemispheric transmission time, cannot be extended to auditory stimuli because there is an almost simultaneous access of auditory information to both hemispheres (Iacoboni and Zaidel, 1999). This finding can be supported by the fact that auditory system has numerous crossover points at various subcortical levels without requiring additional contribution of the callosal commissure, because it seems to occur when subjects with callosal agenesis and early callosotomy have to perform sound localization tasks (Lessard et al., 2002; Hausmann et al., 2005).

Finally, the described approach suggests that advanced MRI tools may help in understanding the neural basis of motor performance deficits when quantitative measures of tissue damage are correlated with appropriate behavioral measurements.

\section{References}

Aboitiz F, Scheibel AB, Fisher RS, Zaidel E (1992) Fiber composition of the human corpus callosum. Brain Res 598:143-153.

Aschersleben G (2002) Temporal control of movements in sensorimotor synchronization. Brain Cogn 48:66-79.

Behrens TE, Woolrich MW, Jenkinson M, Johansen-Berg H, Nunes RG, Clare S, Matthews PM, Brady JM, Smith SM (2003) Characterization and propagation of uncertainty in diffusion-weighted MR imaging. Magn Reson Med 50:1077-1088.

Bove M, Tacchino A, Novellino A, Trompetto C, Abbruzzese G, Ghilardi MF (2007) The effects of rate and sequence complexity on repetitive finger movements. Brain Res 1153:84-91.

Brinkman J, Kuypers HG (1972) Splitbrain monkeys: cerebral control of ipsilateral and contralateral arm, hand, and finger movements. Science 176:536-539.

Brinkman J, Kuypers HG (1973) Cerebral control of contralateral and ipsilateral arm, hand and finger movements in the split-brain rhesus monkey. Brain 96:653-674. 
Caille S, Sauerwein HC, Schiavetto A, Villemure JG, Lassonde M (2005) Sensory and motor interhemispheric integration after section of different portions of the anterior corpus callosum in nonepileptic patients. Neurosurgery 57:50-58; discussion 58-59.

Chepuri NB, Yen YF, Burdette JH, Li H, Moody DM, Maldjian JA (2002) Diffusion anisotropy in the corpus callosum. Am J Neuroradiol 23:803-808.

de Lacoste MC, Kirkpatrick JB, Ross ED (1985) Topography of the human corpus callosum. J Neuropathol Exp Neurol 44:578-591.

Eliassen JC, Baynes K, Gazzaniga MS (1999) Direction information coordinated via the posterior third of the corpus callosum during bimanual movements. Exp Brain Res 128:573-577.

Eliassen JC, Baynes K, Gazzaniga MS (2000) Anterior and posterior callosal contributions to simultaneous bimanual movements of the hands and fingers. Brain 123:2501-2511.

Evangelou N, Konz D, Esiri MM, Smith S, Palace J, Matthews PM (2000) Regional axonal loss in the corpus callosum correlates with cerebral white matter lesion volume and distribution in multiple sclerosis. Brain 123:1845-1849.

Gazzaniga MS (1966) Visuomotor integration in split-brain monkeys with other cerebral lesions. Exp Neurol 16:289-298.

Gazzaniga MS, Freedman H (1973) Observations on visual processes after posterior callosal section. Neurology 23:1126-1130.

Gazzaniga MS, Bogen JE, Sperry RW (1967) Dyspraxia following division of the cerebral commissures. Arch Neurol 16:606-612.

Ge Y, Law M, Johnson G, Herbert J, Babb JS, Mannon LJ, Grossman RI (2004) Preferential occult injury of corpus callosum in multiple sclerosis measured by diffusion tensor imaging. J Magn Reson Imaging 20:1-7.

Ge Y, Law M, Grossman RI (2005) Applications of diffusion tensor MR imaging in multiple sclerosis. Ann NY Acad Sci 1064:202-219.

Geschwind N, Kaplan E (1962) A human cerebral deconnection syndrome. A preliminary report. Neurology 12:675-685.

Hasan KM, Gupta RK, Santos RM, Wolinsky JS, Narayana PA (2005) Diffusion tensor fractional anisotropy of the normal-appearing seven segments of the corpus callosum in healthy adults and relapsing-remitting multiple sclerosis patients. J Magn Reson Imaging 21:735-743.

Hausmann M, Corballis MC, Fabri M, Paggi A, Lewald J (2005) Sound lateralization in subjects with callosotomy, callosal agenesis, or hemispherectomy. Brain Res Cogn Brain Res 25:537-546.

Iacoboni M, Zaidel E (1999) The crossed-uncrossed difference in simple reaction times to lateralized auditory stimuli is not a measure of interhemispheric transmission time: evidence from the split brain. Exp Brain Res 128:421-424.

Jeeves MA, Simpson DA, Geffen G (1979) Functional consequences of the transcallosal removal of intraventricular tumours. J Neurol Neurosurg Psychiatry 42:134-142.

Johansen-Berg H, Della-Maggiore V, Behrens TE, Smith SM, Paus T (2007) Integrity of white matter in the corpus callosum correlates with bimanual co-ordination skills. NeuroImage 36 [Suppl 2]:T16-T21.

Kennerley SW, Diedrichsen J, Hazeltine E, Semjen A, Ivry RB (2002) Callosotomy patients exhibit temporal uncoupling during continuous bimanual movements. Nat Neurosci 5:376-381.

Koechlin E, Jubault T (2006) Broca's area and the hierarchical organization of human behavior. Neuron 50:963-974.

Kurtzke JF (1983) Rating neurologic impairment in multiple sclerosis: an expanded disability status scale (EDSS). Neurology 33:1444-1452.

Larson EB, Burnison DS, Brown WS (2002) Callosal function in multiple sclerosis: bimanual motor coordination. Cortex 38:201-214.

Le Bihan D, Mangin JF, Poupon C, Clark CA, Pappata S, Molko N, Chabriat H (2001) Diffusion tensor imaging: concepts and applications. J Magn Reson Imaging 13:534-546.

Lessard N, Lepore F, Villemagne J, Lassonde M (2002) Sound localization in callosal agenesis and early callosotomy subjects: brain reorganization and/or compensatory strategies. Brain 125:1039-1053.
Marzi CA, Perani D, Tassinari G, Colleluori A, Maravita A, Miniussi C, Paulesu E, Scifo P, Fazio F (1999) Pathways of interhemispheric transfer in normals and in a split-brain subject. A positron emission tomography study. Exp Brain Res 126:451-458.

Miller EK (2000) The prefrontal cortex and cognitive control. Nat Rev Neurosci 1:59-65.

Najenson T, Ron S, Behroozi K (1989) Temporal characteristics of tapping responses in healthy subjects and in patients who sustained cerebrovascular accident. Brain Behav Evol 33:175-178.

Neil JJ, Shiran SI, McKinstry RC, Schefft GL, Snyder AZ, Almli CR, Akbudak E, Aronovitz JA, Miller JP, Lee BC, Conturo TE (1998) Normal brain in human newborns: apparent diffusion coefficient and diffusion anisotropy measured by using diffusion tensor MR imaging. Radiology 209:57-66.

Oh J, Henry RG, Genain C, Nelson SJ, Pelletier D (2004) Mechanisms of normal appearing corpus callosum injury related to pericallosal $\mathrm{T} 1$ lesions in multiple sclerosis using directional diffusion tensor and 1H MRS imaging. J Neurol Neurosurg Psychiatry 75:1281-1286.

Oldfield RC (1971) The assessment and analysis of handedness: the Edinburgh inventory. Neuropsychologia 9:97-113.

Pelletier J, Habib M, Brouchon M, Poncet M, Lyon-Caen O, Salamon G, Khalil R (1992) Interhemispheric transfer in multiple sclerosis. Morphofunctional correlations (in French). Rev Neurol (Paris) 148:672-679.

Pelletier J, Habib M, Lyon-Caen O, Salamon G, Poncet M, Khalil R (1993) Functional and magnetic resonance imaging correlates of callosal involvement in multiple sclerosis. Arch Neurol 50:1077-1082.

Poser CM, Paty DW, Scheinberg L, McDonald WI, Davis FA, Ebers GC, Johnson KP, Sibley WA, Silberberg DH, Tourtellotte WW (1983) New diagnostic criteria for multiple sclerosis: guidelines for research protocols. Ann Neurol 13:227-231.

Preilowski B, ed (1975) Bilateral motor interaction: perceptual motor performance of partial and complete "split brain" patients. Berlin: Springer.

Preilowski BF (1972) Possible contribution of the anterior forebrain commissures to bilateral motor coordination. Neuropsychologia 10:267-277.

Risse GL, Gates J, Lund G, Maxwell R, Rubens A (1989) Interhemispheric transfer in patients with incomplete section of the corpus callosum. Anatomic verification with magnetic resonance imaging. Arch Neurol 46:437-443.

Schnider A, Benson F, Rosner LJ (1993) Callosal disconnection in multiple sclerosis. Neurology 43:1243-1245.

Serrien DJ, Nirkko AC, Wiesendanger M (2001) Role of the corpus callosum in bimanual coordination: a comparison of patients with congenital and acquired callosal damage. Eur J Neurosci 14:1897-1905.

Sidtis JJ, Volpe BT, Holtzman JD, Wilson DH, Gazzaniga MS (1981) Cognitive interaction after staged callosal section: evidence for transfer of semantic activation. Science 212:344-346.

Smith SM, Jenkinson M, Woolrich MW, Beckmann CF, Behrens TE, Johansen-Berg H, Bannister PR, De Luca M, Drobnjak I, Flitney DE, Niazy RK, Saunders J, Vickers J, Zhang Y, De Stefano N, Brady JM, Matthews PM (2004) Advances in functional and structural MR image analysis and implementation as FSL. NeuroImage 23 [Suppl 1]:S208-S219.

Stenneken P, Cole J, Paillard J, Prinz W, Aschersleben G (2003) Coordinating movements in time: the role of sensory movement consequences. J Int Neuropsychol Soc 9:574.

Stenneken P, Prinz W, Cole J, Paillard J, Aschersleben G (2006) The effect of sensory feedback on the timing of movements: evidence from deafferented patients. Brain Res 1084:123-131.

Sternad D, Wei K, Diedrichsen J, Ivry RB (2007) Intermanual interactions during initiation and production of rhythmic and discrete movements in individuals lacking a corpus callosum. Exp Brain Res 176:559-574.

Takahashi CD, Reinkensmeyer DJ (2003) Hemiparetic stroke impairs anticipatory control of arm movement. Exp Brain Res 149:131-140.

Volpe BT, Sidtis JJ, Holtzman JD, Wilson DH, Gazzaniga MS (1982) Cortical mechanisms involved in praxis: observations following partial and complete section of the corpus callosum in man. Neurology 32:645-650. 